\title{
The distance and neutral environment of the massive stellar cluster Westerlund 1
}

\author{
R. Kothes ${ }^{1,2}$ and S. M. Dougherty ${ }^{1}$ \\ 1 National Research Council of Canada, Herzberg Institute of Astrophysics, Dominion Radio Astrophysical Observatory, \\ PO Box 248, Penticton, British Columbia, V2A 6J9, Canada \\ e-mail: roland.kothes@nrc.ca \\ 2 Department of Physics and Astronomy, University of Calgary, 2500 University Drive N.W., Calgary, AB, Canada
}

Received 16 February 2007 / Accepted 11 April 2007

\begin{abstract}
Context. In spite of a large number of recent publications about the massive stellar cluster Westerlund 1, its distance from the Sun remains uncertain with values as low as $1.1 \mathrm{kpc}$, but largely between 4 and $5 \mathrm{kpc}$.

Aims. The goal of this study is to determine a distance to Westerlund 1 independent of the characteristics of the stellar population and to study its neutral environment, using observations of atomic hydrogen.

Methods. The H I observations are taken from the Southern Galactic Plane Survey to study H I absorption in the direction of the $\mathrm{H}$ II region created by the members of Westerlund 1 and to investigate its environment as observed in the H I line emission. A Galactic rotation curve was derived using the recently revised values for the Galactic centre distance of $R_{\odot}=7.6 \mathrm{kpc}$, and the velocity of the Sun around the Galactic centre of $\Theta_{\odot}=214 \mathrm{~km} \mathrm{~s}^{-1}$. This rotation curve successfully predicts the location of the Tangent point gas and the velocity of the Sagittarius Arm outside the solar circle on the far side of the Galaxy to within $4 \mathrm{~km} \mathrm{~s}^{-1}$. Compared to the typically used values of $R_{\odot}=8.5 \mathrm{kpc}$ and $\Theta_{\odot}=220 \mathrm{~km} \mathrm{~s}^{-1}$ this reduces kinematically determined distances by more than $10 \%$.

Results. The newly determined rotation model leads us to derive a distance of $3.9 \pm 0.7 \mathrm{kpc}$ to Westerlund 1 , consistent with a location in the Scutum-Crux Arm. Included in this estimate is a very careful investigation of possible sources of error for the Galactic rotation curve. We also report on small expanding $\mathrm{H}$ I features around the cluster with a maximum dynamic age of 600000 years and a larger bubble which has a minimum dynamic age of 2.5 million years. Additionally we re-calculated the kinematic distances to nearby $\mathrm{H}$ II regions and supernova remnants based on our new Galaxic rotation curve.

Conclusions. We propose that in the early stages of the development of $\mathrm{Wd} 1$ a large interstellar bubble of diameter about $50 \mathrm{pc}$ was created by the cluster members. This bubble has a dynamic age similar to the age of the cluster. Small expanding bubbles, with dynamical ages $\sim 0.6 \mathrm{Myr}$ are found around Wd 1, which we suggest consist of recombined material lost by cluster members through their winds.
\end{abstract}

Key words. Galaxy: open clusters and associations: individual: Westerlund 1 - ISM: bubbles - stars: winds, outflows ISM: H II regions - ISM: supernova remnants

\section{Introduction}

Westerlund 1 (Wd 1) is a highly reddened compact cluster with a large population of post-main sequence massive stars, including OB supergiants and hypergiants, red and yellow supergiants, and Wolf-Rayet (WR) stars (Clark \& Negueruela 2002; Clark et al. 2005). The mass of Wd 1 is likely to be in excess of $10^{5} M_{\odot}$ (Clark et al. 2005), exceeding that of any of the other known massive Galactic clusters, including NGC 6303 (Crowther \& Dessart 1998), the Arches (Figer et al. 2002) and Quintuplet (Figer et al. 1999), and is more comparable to the mass of Super Star Clusters (SSC), previously identified only in other galaxies. If Wd 1 is indeed a Super Star Cluster within our own Galaxy (Clark et al. 2005), this is a unique opportunity to study the properties of a nearby SSC, where it is possible to resolve the individual massive stars, and determine basic properties more readily than in the typically more distant examples.

In spite of a large number of recent observations of Wd 1, its distance from the Sun remains somewhat uncertain. Piatti et al. (1998) derived a distance of $1.1 \pm 0.4 \mathrm{kpc}$ based on photometry of the OB supergiants identified at the time. Using the yellow hypergiants where a spectroscopic luminosity discriminant is available that is lacking for OB supergiants, Clark et al. (2005) determined an upper limit for the distance of $5.5 \mathrm{kpc}$. Clark et al. (2005) also argue for a lower limit on the distance of $2 \mathrm{kpc}$ based on an analysis of radio continuum data (Clark et al. 1998). They also argue that the comparatively low distance of Piatti et al. (1998) is the result of an error in both the absolute magnitude calibration and the reddening law that was used. Additionally, Clark et al. (2005) did not identify any dwarf or giant stars, with all the OB stars being supergiants, unlike the work of Piatti et al. where detection of the main sequence was claimed. Further support for a distance in excess of $2 \mathrm{kpc}$ comes from analysis of near-IR photometry of the WR stars in Wd 1 (Crowther et al. 2006), where the average distance modulus derived from 23 of the 24 known WR stars leads to a distance of $4.7 \pm 1.1 \mathrm{kpc}$. The relatively large uncertainty in this estimate is a result of the uncertainty in the absolute magnitude calibration of WR stars. A more precise estimate of distance comes from an initial analysis of deep IR imaging with the VLT that reveals 
the main sequence and pre-main sequence populations in $\mathrm{Wd} 1$ from which a photometric distance of $4.0 \pm 0.3 \mathrm{kpc}$ is deduced (Brandner et al. 2005).

The most recent analyses appear to be converging on a distance in the range 4-5 kpc. In this paper, we examine $\mathrm{H} \mathrm{I}$ data in the direction of Wd 1 from the Southern Galactic Plane Survey (SGPS) (McClure-Griffiths et al. 2005) to estimate a distance to Wd 1 based on HI absorption and related Hi features. We also examine the large-scale distribution of $\mathrm{H} \mathrm{I}$ in the environment of Wd 1 to search for evidence of the impact of the putative SSC on the surrounding interstellar medium.

\section{The SGPS HI data set}

The Southern Galactic Plane Survey (McClure-Griffiths et al. 2005) is part of the International Galactic Plane Survey (IGPS), a project devoted to mapping the neutral hydrogen in the plane of the Galaxy, the Milky Way, at arcminute scale. The SGPS maps the region of the Milky Way visible in the southern hemisphere. Other parts of the IGPS are the Canadian Galactic Plane Survey (CGPS, Taylor et al. 2003), which covers the Galactic plane seen in the northern hemisphere, and the VLA Galactic Plane Survey (VGPS, Stil et al. 2006), which connects the two other surveys through the first quadrant of the Milky Way.

The SGPS data were obtained with the Australia Telescope Compact Array (ATCA) and the Parkes 64-m radio telescope. The survey covers an area of $325^{\circ}$ from $253^{\circ}$ to $358^{\circ}$ and $5^{\circ}$ to $20^{\circ}$ in Galactic longitude and from -1.5 to $+1^{\circ} .5$ in Galactic latitude. In the direction of $\mathrm{Wd} 1$ the total velocity coverage is $600 \mathrm{~km} \mathrm{~s}^{-1}$ from -300 to $+300 \mathrm{~km} \mathrm{~s}^{-1}$. The angular resolution is $\sim 2^{\prime}$ and the sensitivity $\sim 1.6 \mathrm{~K}$.

\section{Results}

\subsection{Galactic kinematics in the direction of Westerlund 1}

We carefully investigated the dynamics along the line of sight towards Wd 1. As a starting point we used a flat rotation model to describe the circular rotation in our Galaxy. For this model we have to determine the Galactocentric radius of the Sun $R_{\odot}$ and its circular velocity around the Galactic centre $\Theta_{\odot}$. Instead of assuming the standard values, $R_{\odot}=8.5 \mathrm{kpc}$ and $\Theta_{\odot}=220 \mathrm{~km} \mathrm{~s}^{-1}$, which are still used in most kinematic distance estimates and Galactic models (e.g. Cordes \& Lazio 2002), we carefully investigated the literature to find the most recent estimates. $R_{\odot}$ was determined to be $7.6 \pm 0.3 \mathrm{kpc}$ by Eisenhauer et al. (2005) from a high precision measurement of the three dimensional orbit of the star S2, which is orbiting the black hole at the centre of the Galaxy at a distance of less than $0.2^{\prime \prime}$. This is significantly smaller $(\sim 11 \%)$ than the previously assumed distance. Feast \& Whitelock (1997) determined the angular velocity of circular rotation at the position of the Sun $\Omega_{\odot}$, from the Oort constants using data from the Hipparcos satellite. $\Omega_{\odot}=\frac{\Theta_{\odot}}{R_{\odot}}=27.2 \pm$ $0.8 \mathrm{~km} \mathrm{~s}^{-1} \mathrm{kpc}^{-1}$ gives $\Theta_{\odot}=207 \pm 10 \mathrm{~km} \mathrm{~s}^{-1}$. Another technique to determine $\Theta_{\odot}$ is the proper motion of the Galactic Centre. Reid \& Brunthaler (2004) found a proper motion of

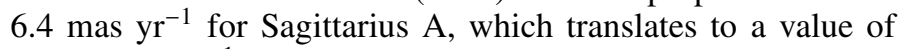
$229 \pm 10 \mathrm{~km} \mathrm{~s}^{-1}$ for a distance of $7.6 \mathrm{kpc}$ to the Galactic Centre. Correcting this for the peculiar motion of the Sun, which Reid $\&$ Brunthaler (2004) suggest is between 5 and $12 \mathrm{~km} \mathrm{~s}^{-1}$, we derive $\Theta_{\odot}=220 \pm 11 \mathrm{~km} \mathrm{~s}^{-1}$. Both values agree within their uncertainties, and averaging the two values we obtain $\Theta_{\odot}=214 \pm$ $7 \mathrm{~km} \mathrm{~s}^{-1}$. With these values for $R_{\odot}$ and $\Theta_{\odot}$ we can derive the

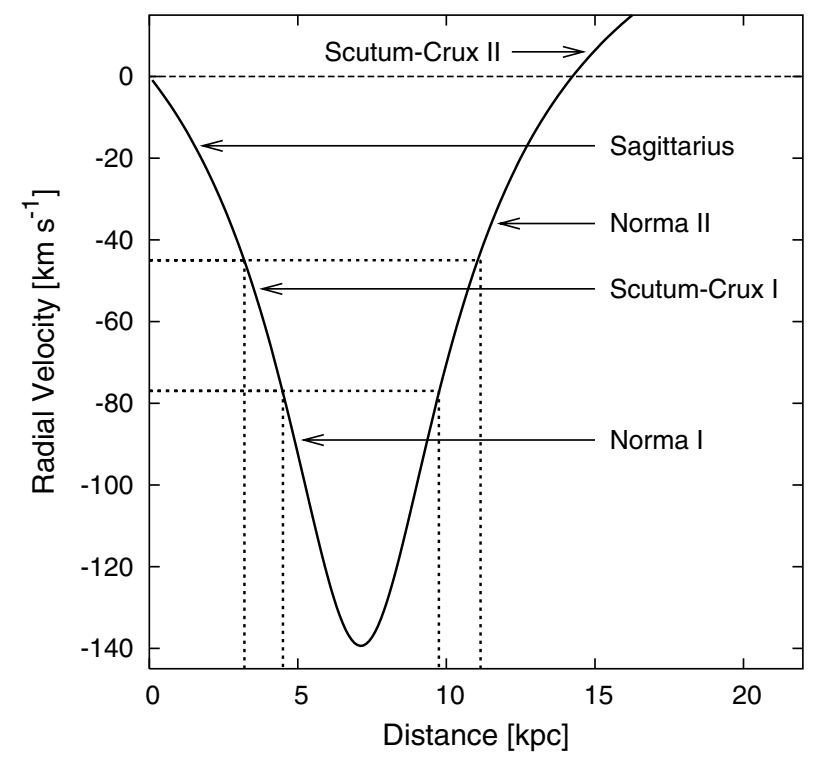

Fig. 1. The Galactic rotation curve toward Galactic longitude of $339^{\circ} .6$ using a Galactocentric radius of $R_{\odot}=7.6 \mathrm{kpc}$ and a circular velocity of $\Theta_{\odot}=214 \mathrm{~km} \mathrm{~s}^{-1}$ for the Sun. We assumed a flat rotation curve and purely circular rotation. The approximate locations of the spiral arms in radial velocity are indicated. Here the indices I and II indicate the first and second time the line of sight passes through that spiral arm (see also Fig. 2).

systemic velocity $v_{\text {sys }}$ (local standard of rest velocity) measured from the Sun in the direction of $\mathrm{Wd} 1$ as a function of distance (Fig. 1).

We tested our assumption of a flat rotation curve by comparing the measured Tangent point velocity from the SGPS data with the velocity extrapolated from our values for $\Theta_{\odot}$. The Tangent point at any line of sight through the inner Galaxy is the point at which we look tangential to the circular motion and hence find the largest line of sight velocity. For flat rotation we should observe a radial velocity of $-139 \mathrm{~km} \mathrm{~s}^{-1}$ at the Tangent point in the direction of $l=339.6^{\circ}$ (Fig. 1). This agrees reasonably well with the observed value of somewhere between $-130 \mathrm{~km} \mathrm{~s}^{-1}$ and $-135 \mathrm{~km} \mathrm{~s}^{-1}$ (Fig. 3). This is actually a quite remarkable result, since the Tangent point in the direction of $\mathrm{Wd} 1$ is at a distance of about $7 \mathrm{kpc}$ from the Sun at a Galacto-centric radius of about $2.6 \mathrm{kpc}$. This should be well within the area which is heavily influenced by the central bar of the Milky Way. Benjamin et al. (2005) determined that the central bar has a maximum distance of $4.4 \mathrm{kpc}$ from the Galactic centre, using a Galacto-centric distance of $8.5 \mathrm{kpc}$ for the Sun. With the value of $R_{\odot}=7.6 \mathrm{kpc}$ this would translate to a maximum distance of $3.9 \mathrm{kpc}$ which is significantly higher than the Galactoc-centric distance of the Tangent point.

To determine the distance to the spiral arms in the direction of $\mathrm{Wd} 1$ we utilise the distribution of free electrons in our Galaxy as described in the models of Taylor \& Cordes (1993) and Cordes \& Lazio (2002). The peaks in electron density indicate the locations of the spiral arms in this direction of the Galaxy (Fig. 2). Since Taylor \& Cordes (1993) and Cordes \& Lazio (2002) used a Galacto-centric radius of $R_{\odot}=8.5 \mathrm{kpc}$ for the Sun in their spiral arm model, we re-scaled this profile for $R_{\odot}=7.6 \mathrm{kpc}$. The distances to the nearby $\mathrm{H}$ II regions used to define the distance of the nearby spiral arms were derived spectroscopically. Hence, we left the distance to the two nearby spiral arms alone and only re-scaled the distances to the other more 


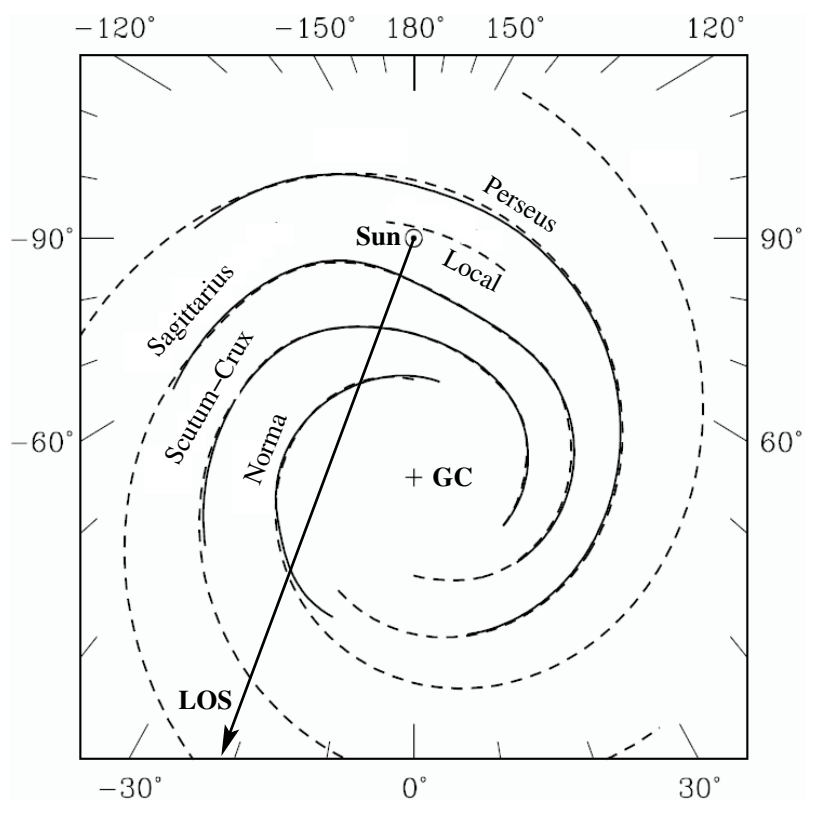

Fig. 2. The spiral arm pattern of the Milky Way Galaxy (Taylor \& Cordes 1993; Cordes \& Lazio 2002). The names of the spiral arms and the line of sight (LOS) toward Wd 1 are indicated.

Table 1. The systemic velocity and distance to the spiral arms along the line of sight toward Wd 1 determined with the Spiral arm model in Fig. 2 and the rotation curve in Fig. 1.

\begin{tabular}{lcc}
\hline \hline Spiral Arm & $v_{\text {sys }}\left[\mathrm{km} \mathrm{s}^{-1}\right]$ & $d_{\text {lit }}[\mathrm{kpc}]$ \\
\hline Sagittarius I & -17 & 1.5 \\
Scutum-Crux I & -52 & 3.5 \\
Norma I & -89 & 4.9 \\
Norma II & -35 & 11.5 \\
Scutum-Crux II & +6 & 15.0 \\
Sagittarius II & +26 & 18.4 \\
\hline
\end{tabular}

distant spiral arms. With the rotation curve in Fig. 1, we derive the systemic velocities at the centres of the individual spiral arms. These newly determined distances and systemic velocities are listed in Table 1. A comparison of these values with the $\mathrm{HI}$ emission profile in the direction of $\mathrm{Wd} 1$ is shown in Fig. 3.

The locations of the individual spiral arms in velocity space agree remarkably well with the brightness temperature peaks in the HI emission profile (Fig. 3). The emission peak between -140 and $-100 \mathrm{~km} \mathrm{~s}^{-1}$ near the Tangent point is produced by a pile up in velocity space rather than a real density enhancement. In this region, the velocity does not change significantly with distance, resulting in one velocity channel containing the emission from $\mathrm{HI}$ distributed over a much larger distance interval than in other locations along the line of sight. This creates a peak in the HI emission profile close to the Tangent point velocity. There are two spiral arms that can be seen distinct from the others, Norma I with a centre velocity of $-89 \mathrm{~km} \mathrm{~s}^{-1}$ and Sagittarius II, for which the model centre velocity of $+26 \mathrm{~km} \mathrm{~s}^{-1}$ is very close to the emission peak at $+22 \mathrm{~km} \mathrm{~s}^{-1}$. Since Sagittarius II is supposed to be well outside the Solar Circle on the other side of the Galaxy in any Galactic model, this agreement with the H I emission profile is incredible and boosts our confidence in our rotation model. The other spiral arms are clumped into two groups: Scutum-Crux I and Norma II merge at a radial velocity of about $-40 \mathrm{~km} \mathrm{~s}^{-1}$ and the Local arm, Sagittarius I, and Scutum-Crux II are at low negative velocities.

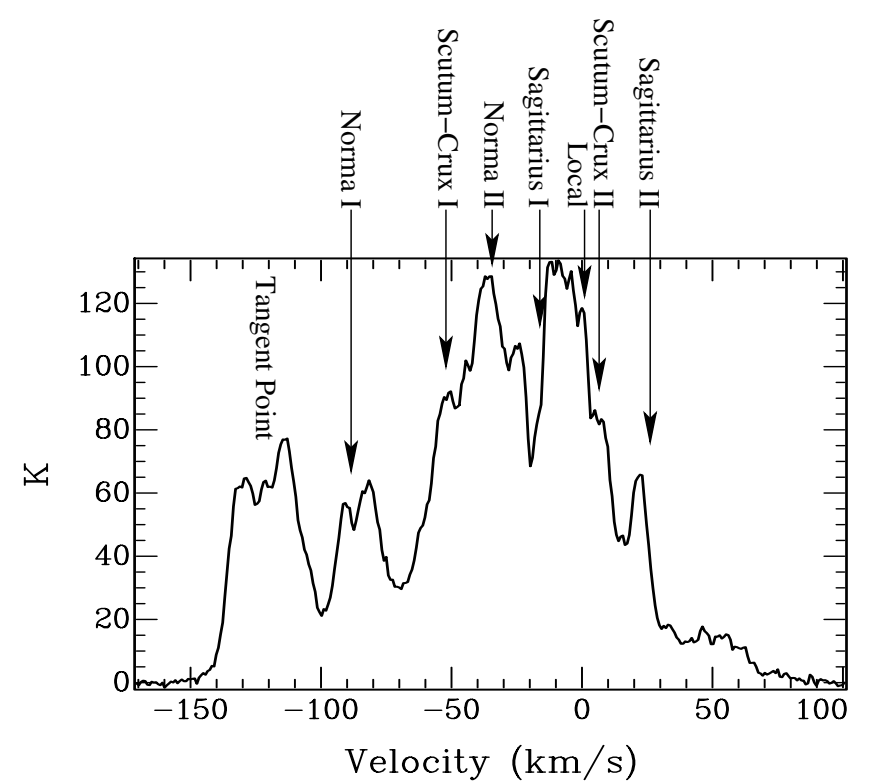

Fig. 3. H I emission profile averaged over an area of a few arcminutes towards $\mathrm{Wd} 1$. The locations of the spiral arms as listed in Table 1 are indicated. The Local arm is indicated at $0 \mathrm{~km} \mathrm{~s}^{-1}$.

\subsection{HI absorption}

The radio continuum emission of the $\mathrm{H}$ II region produced by the Wd 1 cluster is not bright enough at $1420 \mathrm{MHz}$ to produce a prominent $\mathrm{H}$ I absorption profile. Its peak brightness temperature is only about $24 \mathrm{~K}$ in the SGPS data and the source is barely resolved. Its diameter is about $3^{\prime}$ at a resolution of $2^{\prime}$. Therefore, we have to average over several velocity channels to amplify the weak absorption signal and increase its signalto-noise ratio. For each spiral arm along the line of sight we tried to average those channels that are likely to produce the deepest absorption. In these absorption profiles we can identify those velocity intervals in the spiral arms where the H I produces deep and distinct absorption features and those that do not. As a reference we used the absorption profiles of the nearby $\mathrm{H}$ II regions shown in Fig. 4. Two of these, representing the G338.9+0.6 and the G340.2-0.4 complexes, are Scutum-Crux I objects according to their systemic velocity (see Table 2 ). The other two H II regions are located beyond the Tangent point in Norma II (G338.4+0.1 complex) and G338.41-0.24 might even reside in Scutum-Crux II.

The absorption of the three nearby arms, the Local arm, Sagittarius I, and Scutum-Crux I, was identified in the absorption profiles of the two H II regions in Scutum-Crux I since their profiles are not contaminated by absorption of $\mathrm{H}$ I located beyond the Tangent point. For Norma I and the Tangent point we used the two H II regions that are located beyond the Tangent point on the other side of the Galaxy. We found distinct absorption in the Local arm between +5 and $-1 \mathrm{~km} \mathrm{~s}^{-1}$, for Sagittarius I between -10 and $-20 \mathrm{~km} \mathrm{~s}^{-1}$, for Scutum-Crux I between -32 and $-45 \mathrm{~km} \mathrm{~s}^{-1}$, for Norma I between -85 and $-102 \mathrm{~km} \mathrm{~s}^{-1}$, and for the Tangent point between -110 and $-125 \mathrm{~km} \mathrm{~s}^{-1}$. For ScutumCrux I we did not use negative velocities higher than $-45 \mathrm{~km} \mathrm{~s}^{-1}$ to avoid confusion with a possible bubble centered at Wd 1 (see Sect. 3.3).

The results of our averaging procedure are shown in Fig. 5. It is apparent that the radio continuum emission from $\mathrm{Wd} 1$ is absorbed by the Local arm, Sagittarius I, and Scutum-Crux I. This is indicated by a hole in the distribution of $\mathrm{HI}$ emission at the 


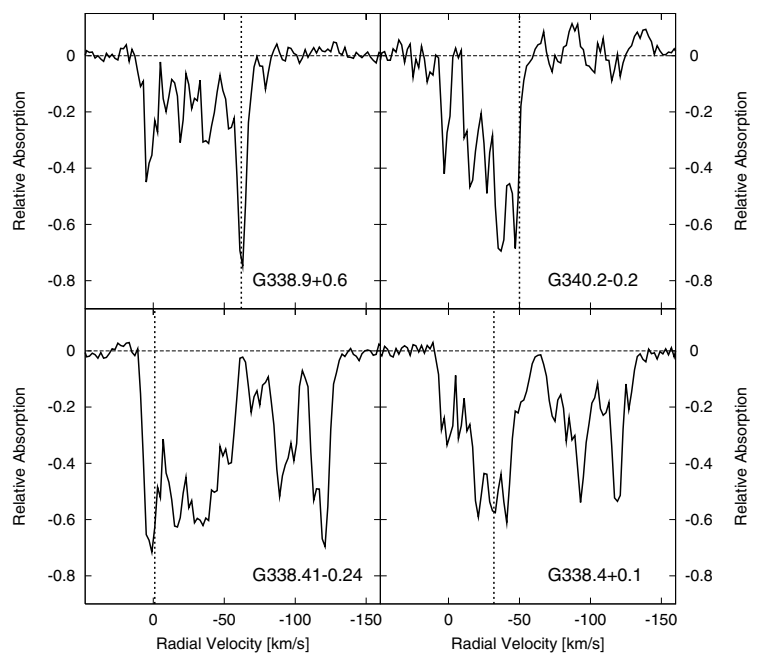

Fig. 4. H I absorption profiles of four radio bright $\mathrm{H}$ II regions in the vicinity of $\mathrm{Wd} 1$. To make the individual components better comparable we plotted the relative absorption - the ratio of absorbed emission to total emission - as a function of radial velocity. The dotted vertical lines indicate the systemic velocities of the H II regions (Russeil 2003), which, within their uncertainties, are in excellent agreement with the absorption peaks.

Table 2. Characteristics of radio bright $\mathrm{H}$ II regions and supernova remnants in the vicinity of Wd 1 . Column 1 contains a name based on the Galactic coordinates of the object. Columns 2 and 3 contain information about the systemic velocity and derived kinematic distance found in the literature (Russeil 2003). Column 4 contains the distance to the source based on the rotation curve and method described in Sects. 3.1 and 4.1, respectively. Column 5 indicates the proposed spiral arm the object resides in.

\begin{tabular}{lcccl}
\hline \hline Source & $\begin{array}{c}v_{\text {sys }} \\
{\left[\mathrm{km} \mathrm{s}^{-1}\right]}\end{array}$ & $\begin{array}{c}d_{\text {lit }} \\
{[\mathrm{kpc}]}\end{array}$ & $\begin{array}{c}d_{\text {new }} \\
{[\mathrm{kpc}]}\end{array}$ & Spiral Arm \\
\hline G337.8-0.1 & & & $\approx 11$ & Norma II \\
G337.95-0.48 & -41 & 3.1 & $2.9_{-0.4}^{+1.2}$ & Scutum Crux I \\
G338.0-0.1 & -51 & 12.0 & $10.7_{-0.4}^{+1.4}$ & Norma II \\
G338.41-0.24 & -1 & 15.7 & $14.0_{-0.9}^{+4.5}$ & Scutum-Crux II \\
G338.4+0.1 & -32 & 13.1 & $11.7_{-0.5}^{+2.0}$ & Norma II \\
G338.8+0.6 & -62 & 4.3 & $3.9_{-0.4}^{+0.9}$ & Scutum-Crux I \\
G338.5+0.1 & & & $\approx 11$ & Norma II \\
G338.9-0.1 & -38 & 3.1 & $2.8_{-0.6}^{+1.2}$ & Scutum-Crux I \\
G338.9+0.4 & & & $\approx 3.9$ & Scutum-Crux I \\
G339.13-0.41 & -38 & 3.1 & $2.8_{-0.6}^{+1.2}$ & Scutum-Crux I \\
Wd 1 & -55 & & $3.6_{-0.4}^{+1.0}$ & Scutum-Crux I \\
G339.58-0.12 & -34 & 2.8 & $2.6_{-0.7}^{+1.3}$ & Scutum-Crux I \\
G339.84+0.27 & -20 & 14.1 & $12.5_{-0.6}^{+2.7}$ & Norma II \\
G340.2-0.2 & -50 & 3.7 & $3.5_{-0.5}^{+1.0}$ & Scutum-Crux I \\
G340.24-0.48 & -61 & 4.4 & $3.9_{-0.3}^{+0.9}$ & Scutum-Crux I \\
G340.6+0.3 & & & $\approx 15$ & Scutum-Crux II \\
\hline
\end{tabular}

position of Wd 1. There is no evidence for absorption in Norma I or the Tangent point gas. This indicates a location in ScutumCrux I or at the near edge of Norma I. The latter, however, is rather unlikely, because there must not be any absorbing Norma I material between us and Wd 1. All H II regions shown in Fig. 4 have a very deep distinct absorption feature at their systemic velocity, which is likely created by material in their vicinity in the cloud complex from which the stars in those $\mathrm{H}$ II regions were formed. Since the remains of these clouds are expected to be very dense and cold the optical depth should be rather high.

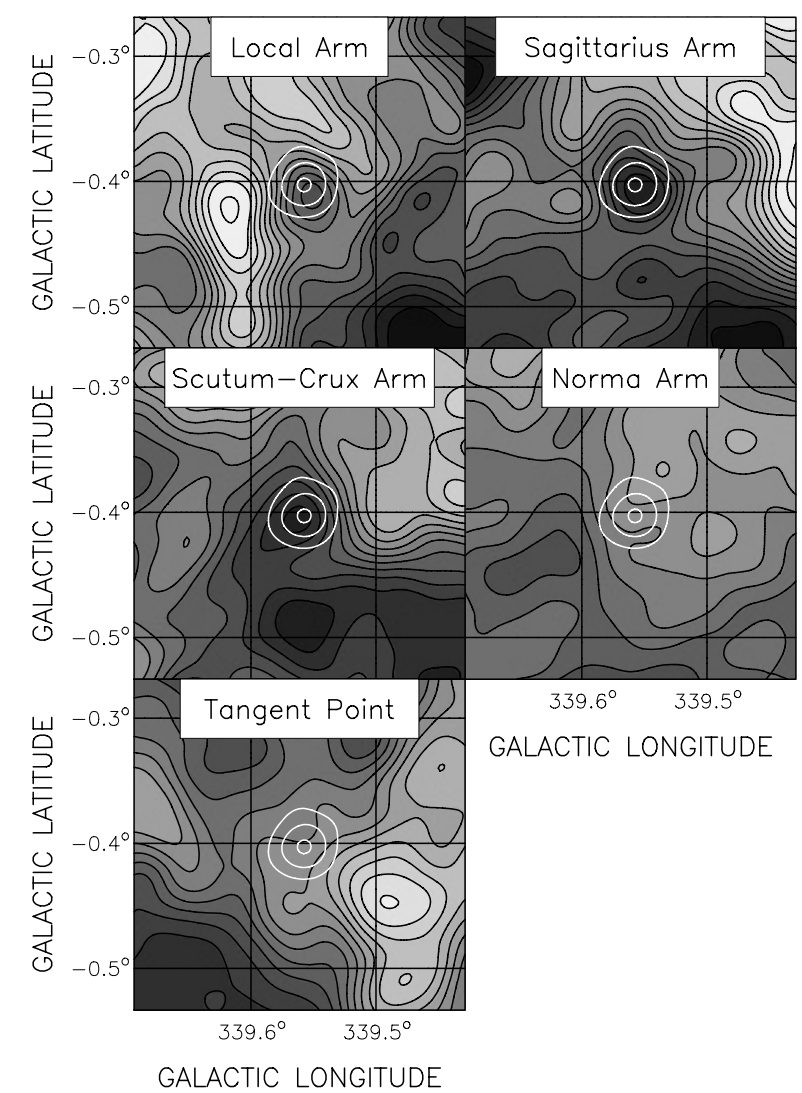

Fig. 5. H I channels images averaged together to demonstrate in which spiral arms Wd 1 is absorbed. For the individual arms we averaged over: +5 to $-1 \mathrm{~km} \mathrm{~s}^{-1}$ (Local arm), -10 to $-20 \mathrm{~km} \mathrm{~s}^{-1}$ (Sagittarius arm), -32 to $-45 \mathrm{~km} \mathrm{~s}^{-1}$ (Scutum-Crux arm), -85 to $-102 \mathrm{~km} \mathrm{~s}^{-1}$ (Norma arm), and -110 to $-125 \mathrm{~km} \mathrm{~s}^{-1}$ (Tangent Point). In these images white denotes high and black weak emission. The radio continuum emission of $\mathrm{Wd} 1$ is indicated by the white contours.

This should produce deep absorption lines. If $\mathrm{Wd} 1$ is located in the Norma arm it cannot have such a component. This makes a Scutum-Crux arm location more likely, however, it does not entirely exclude the possibility of a location at the near edge of the Norma arm.

\subsection{The neutral environment of $W d 1$}

We carefully investigated the HI data in the velocity range of the Scutum-Crux arm and at velocities corresponding to the near edge of the Norma arm. We found one prominent feature in velocity space which is exactly centered at the position of $\mathrm{Wd} 1$ at a velocity of $-55 \mathrm{~km} \mathrm{~s}^{-1}$. This feature is shown in Fig. 6, indicated by the dashed ellipses (top and right panels). The dashed circle in the centre image marks the location of this expanding bubble projected back to the map plane. This feature, B1, cannot be an absorption feature since the depression visible at the location of $\mathrm{Wd} 1$ is deeper than the peak brightness of $\mathrm{Wd} 1$ 's radio continuum emission. In the absorption map of the Scutum-Crux arm (Fig. 5) the velocity interval related to this expanding bubble was omitted to avoid confusion with a hole in the H I map that is created by a lack of $\mathrm{H} \mathrm{I}$ and not actual H I absorption.

Feature B1 seems to be located at the edge of a much larger elliptical bubble (B2), indicated by the dotted ellipses in Fig. 6 . This feature is very obvious in the map plane but is not as clear in velocity space (left and bottom panels of Fig. 6). There are fingers of emission emerging from the bright Scutum-Crux I gas 


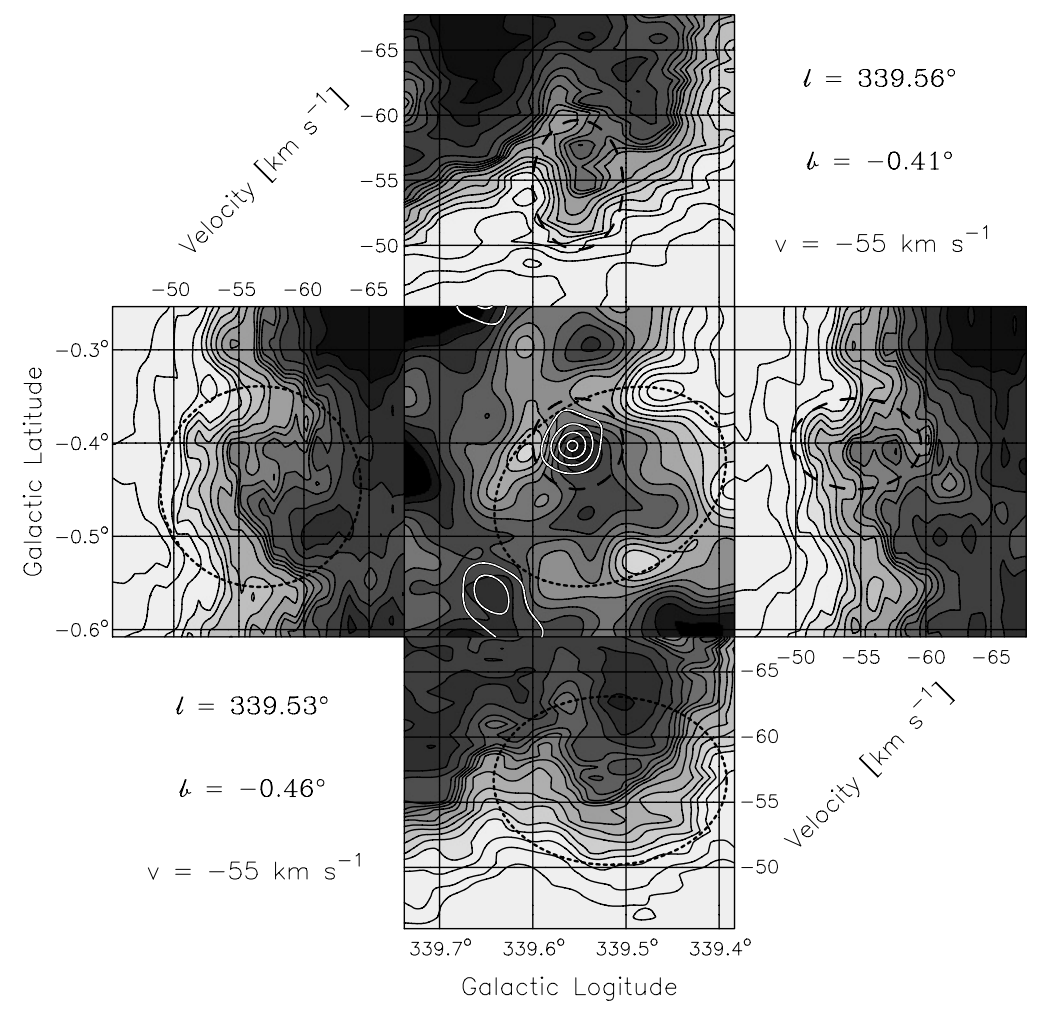

Fig. 6. Three views of the two small expanding bubbles, B1 and B2, around $\mathrm{Wd} 1$, indicated by dashed and dotted ellipses respectively. Longitudevelocity slices are displayed at the top and bottom and the velocity-latitude slices in the left and right panels. The HI channel map at about $-55 \mathrm{~km} \mathrm{~s}^{-1}$ in longitude-latitude is shown in the centre, with Wd 1 indicated by the white contours. The bright emission in the velocity slices mark the gas at the far edge of Scutum-Crux I. In these images black represents low and white high intensity.

towards higher negative velocities, indicating an expansion for this feature. However, these fingers are not closed in an end cap, which would mark the part of the bubble that is moving towards us. Bubble B1 is closed in velocity space, which makes it very easy to detect. The expansion velocity of $\mathrm{B} 1$ is $5 \mathrm{~km} \mathrm{~s}^{-1}$, as readily seen in Fig. 6. The expansion velocity of B2 is not easy to identify since the end cap is missing, but the velocity fingers seem to indicate a somewhat higher expansion velocity. The spatial coincidence of the two features suggests both are associated with Wd 1 , supported by their central velocity of $-55 \mathrm{~km} \mathrm{~s}^{-1}$. This velocity is well within the velocity interval predicted for Wd 1 by the H I absorption measurements. Therefore we adopt a radial velocity of $-55 \pm 3 \mathrm{~km} \mathrm{~s}^{-1}$ for $\mathrm{Wd} 1$. Uncertainties for the radial velocities along the line of sight are discussed in Sect. 4.1.

At a radial velocity of $-55 \mathrm{~km} \mathrm{~s}^{-1}$ a larger field of view reveals a much larger bubble-like feature (B3), which is open to the south, away from the Galactic plane (Fig. 7). The emission associated with Bubble B2 can be seen in the lower right corner of B3. To the north, B3 consists of a shell with two large, bright, and complex emission regions to the east and west. The eastern region contains the $\mathrm{H}$ II complex G340.2-0.2, with a radial velocity of $-50 \mathrm{~km} \mathrm{~s}^{-1}$ (Table 2). This suggests that Wd 1 and the HII region complex G340.2-0.2 are evolving in the same environment, at the far side of Scutum-Crux I.

\section{Discussion}

\subsection{The distance to Westerlund 1}

We determine a radial velocity of $-55 \pm 3 \mathrm{~km} \mathrm{~s}^{-1}$ for $\mathrm{Wd} 1$ from the central velocity of the bubbles in which we believe it

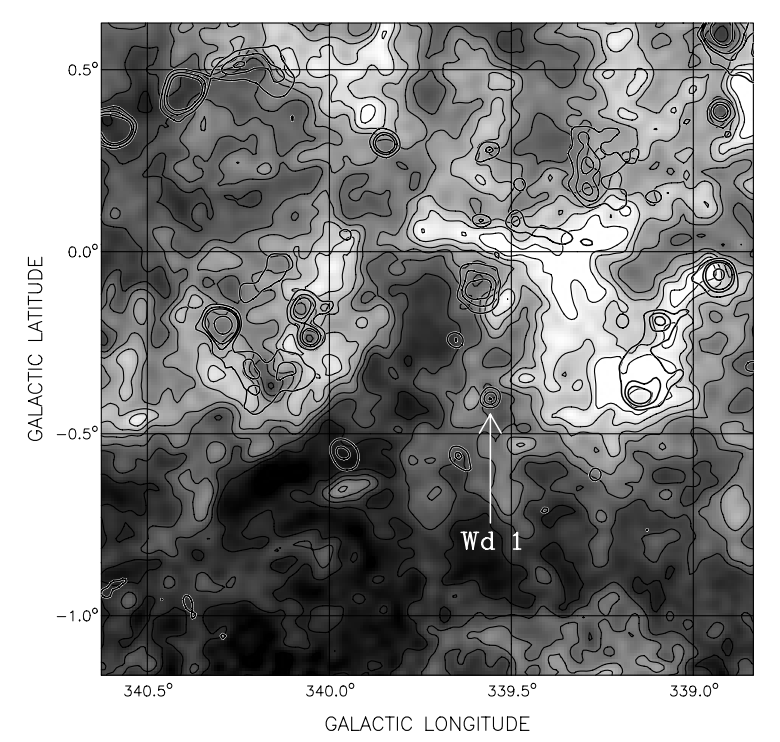

Fig. 7. H I channelmap at a radial velocity of $-55 \mathrm{~km} \mathrm{~s}^{-1}$ from the SGPS. Black contours go from $20 \mathrm{~K}$ to $90 \mathrm{~K}$ in steps of $10 \mathrm{~K}$. Grayish contours indicate the $1420 \mathrm{MHz}$ continuum emission. The location of $\mathrm{Wd} 1$ is indicated.

is located. Using the rotation curve determined in Sect. 3.1 (see Fig. 1) this translates to a distance of $3.6 \pm 0.2 \mathrm{kpc}$ or $10.6 \pm$ $0.2 \mathrm{kpc}$. The closer distance is preferable, because the H II region of $\mathrm{Wd} 1$ does not indicate any absorption in the $\mathrm{HI}$ gas of Norma I or near the Tangent point. Additionally, the previous distance estimates are $\sim 4-5 \mathrm{kpc}$. A comparison with the spiral arm model in Fig. 2 and the calculated distances to the spiral 


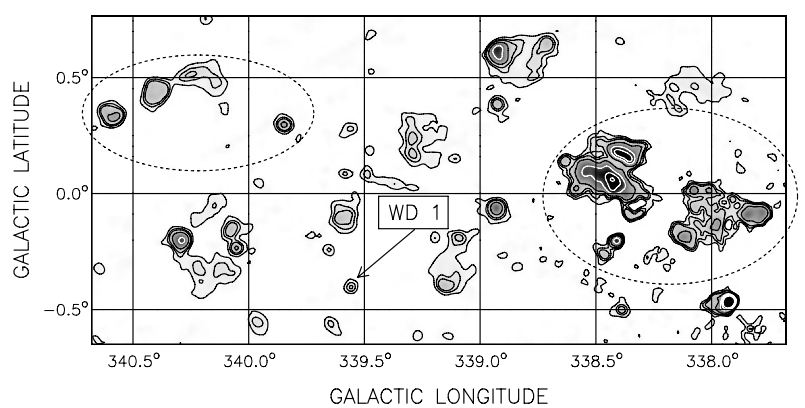

Fig. 8. Radio continuum emission at $1420 \mathrm{MHz}$ around $\mathrm{Wd} 1$ taken from the end channels of the SGPS H I observations. Contours are at 11, 17,23 , and $40 \mathrm{~K}$ (black) and 80, 120, and $160 \mathrm{~K}$ (white). The dotted ellipses enclose SNRs and HII regions that are believed to be beyond the Tangent point. The location of $\mathrm{Wd} 1$ is indicated.

arms in Table 1 reveals that $\mathrm{Wd} 1$ is located in the Scutum-Crux I arm.

A possible source of uncertainty for our rotation curve could be the presence of non-circular motion. Non-circular velocity components due to streaming motion can reach a maximum of about $\pm 10 \mathrm{~km} \mathrm{~s}^{-1}$ (Burton 1972). This would result in $\pm 8 \mathrm{~km} \mathrm{~s}^{-1}$ projected to the line of sight towards $\mathrm{Wd} 1$ assuming a distance of $3.6 \mathrm{kpc}$. Another source of non-circular motion could be a spiral shock, which is always directed towards the Galactic centre. At the location of Wd 1 the spiral shock would be directed away from us, leading to an underestimate of the actual distance. Typical values for the velocity shift due to the spiral shock could reach a maximum of $30 \mathrm{~km} \mathrm{~s}^{-1}$ (Foster \& MacWilliams 2006), which would translate to about $24 \mathrm{~km} \mathrm{~s}^{-1}$ projected to the line of sight. Random motion add another $\pm 5 \mathrm{~km} \mathrm{~s}^{-1}$ to our uncertainty. Taking all these uncertainties into account, we derive a systemic velocity of $-55_{-26}^{+9} \mathrm{~km} \mathrm{~s}^{-1}$ for $\mathrm{Wd} 1$. In the direction of $\mathrm{Wd} \mathrm{1,the}$ velocity versus distance gradient is rather steep (see Fig. 1), so the large velocity uncertainty does not translate to a large distance uncertainty. This radial velocity corresponds to a distance of $3.6_{-0.4}^{+1.0} \mathrm{kpc}$, or by choosing the centre of the uncertainty interval as the most probable distance, we get $3.9 \pm 0.7 \mathrm{kpc}$. This result agrees very well with the latest independent distance estimates of $4.0 \pm 0.3 \mathrm{kpc}$ (Brandner et al. 2005) and $4.7 \pm 1.1 \mathrm{kpc}$ (Crowther et al. 2006). This distance is consistent with a location at the far side of the Scutum-Crux I arm. Furthermore, the bubbles B1 and B2 in Fig. 6 seem to be emerging from the bright gas at the high velocity edge of the Scutum-Crux I arm towards higher negative velocities, supporting a location on the far side of this arm.

\subsection{Distance estimates for HII regions and SNRs in the vicinity of $\mathrm{Wd} 1$}

In Fig. 8 we show a $1420-\mathrm{MHz}$ radio continuum image of the area around $\mathrm{Wd} \mathrm{1,} \mathrm{created} \mathrm{from} \mathrm{the} \mathrm{end-channels} \mathrm{of} \mathrm{the} \mathrm{H} \mathrm{I} \mathrm{data}$ that do not contain any neutral hydrogen emission. Wd 1 is located in a rich area of radio bright $\mathrm{H}$ II regions and supernova remnants. In Table 2 we list $\mathrm{H}$ II regions and complexes for which the systemic velocity is known and for which the distance ambiguity in the inner Galaxy, where each radial velocity corresponds to two possible distances, was solved (Russeil 2003). With the rotation curve shown in Fig. 1, new distances to these objects can be deduced. A comparison of the systemic velocities of these H II regions with the centre velocity of each spiral arm, determined in Sect. 3.1 (see Table 1), gives the most likely spiral arm where these objects reside (Table 2). Almost all of these

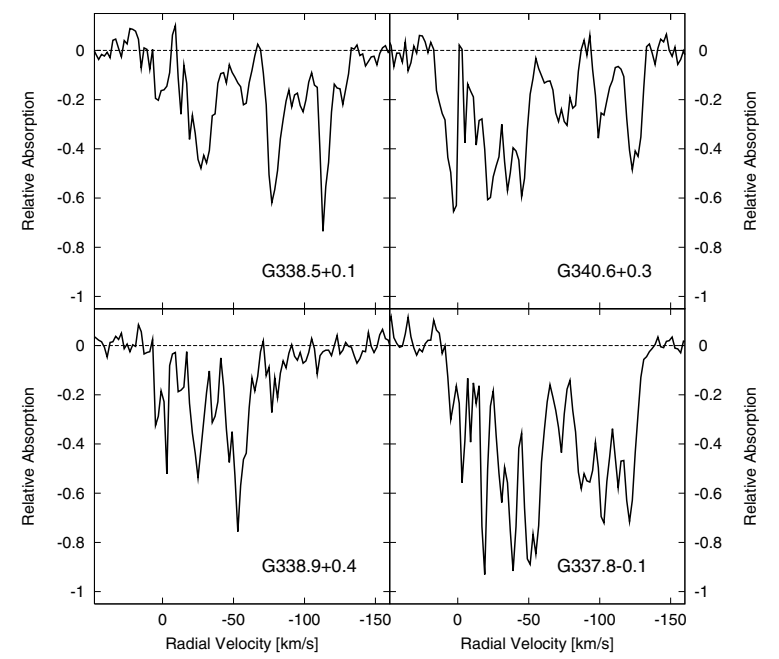

Fig. 9. H I absorption profiles of one radio bright $\mathrm{HII}$ region (G338.9+0.4) and three bright SNRs in the vicinity of $\mathrm{Wd} 1$.

sources are apparently concentrated in the Scutum-Crux I arm and the Norma II arm. Only G338.41 - 0.24 seems to be located even further away in the Scutum-Crux II arm. Objects believed to be beyond the Tangent point are encircled by dashed ellipses in Fig. 8. It is quite curious that no objects were found in either Sagittarius I or Norma I arms.

HI absorption profiles for four additional radio bright sources can also be determined (Fig. 9). The distances and systemic velocities of these objects were previously unknown. These absorption profiles were compared with those in Fig. 4 to determine the most likely spiral arm location for these objects. We then assume the distance to the centre of that particular spiral arm to be the most likely value for these objects, with uncertainties $\sim \pm 1$ kpc (Table 2).

The absorption profile of the HII region G338.9+0.4 in Fig. 9 is very similar to G338.9+0.6 and G340.2-0.2 (see Fig. 4) with no evidence of absorption in Norma I or at the Tangent point. This suggests G338.9 + 0.4 could be a part of the G338.9+0.6 $\mathrm{H}$ II region complex, just to the north (see Fig. 8). Therefore, we propose a Scutum-Crux I location at a distance of about $3.9 \mathrm{kpc}$.

The SNR G340.6+0.3 is absorbed by Norma I and the Tangent point gas and also shows a deep absorption feature with a relative absorption of almost 0.7 at about $0 \mathrm{~km} \mathrm{~s}^{-1}$ (Fig. 9). This makes its absorption profile very similar to that of G338.41-0.24 (Fig. 4). No other source shows such a deep and wide absorption feature around a velocity of $0 \mathrm{~km} \mathrm{~s}^{-1}$. This indicates a location on the other side of the Galaxy in Scutum-Crux II at about $15 \mathrm{kpc}$. This gives the SNR a diameter of about $26 \mathrm{pc}$. It is not unusual that absorption features that are far away seem to be deeper than those that are nearby. The reason for this is simply the area probed by the observing beam becomes bigger with distance so that more $\mathrm{HI}$ is detected within one beam.

The SNRs G337.8-0.1 and G338.5+0.1 show absorption by Norma I and at the Tangent point but lack the deep and wide absorption feature at $0 \mathrm{~km} \mathrm{~s}^{-1}$. Their absorption profiles look very similar to that of G338.4+0.1 in Fig. 4. Therefore these two SNRs are most likely Norma II objects at a distance of about $11 \mathrm{kpc}$. This gives G338.5+0.1 a diameter of about $30 \mathrm{pc}$ and G337.8-0.1 an extent of about $30 \times 20 \mathrm{pc}$. 


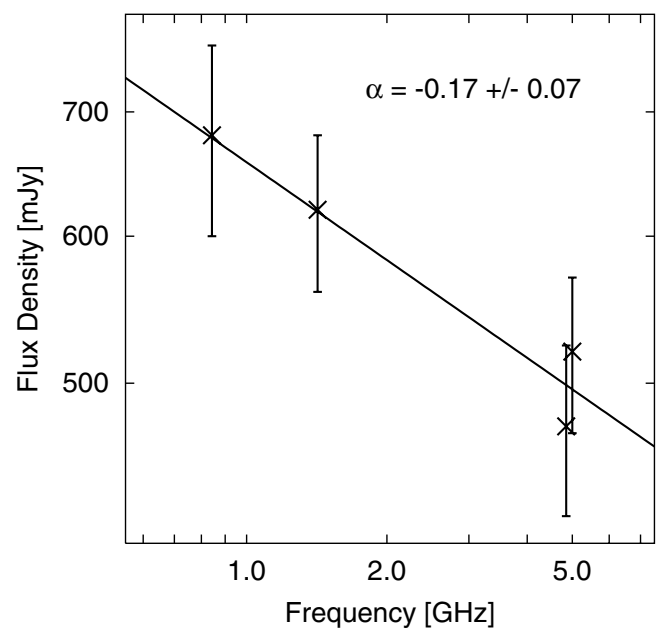

Fig. 10. Radio continuum spectrum of the H II region, produced by the members of Wd 1 .

\subsection{The Wd 1 HII region}

We determined radio continuum flux densities for the $\mathrm{Wd} 1$ $\mathrm{H}$ II region at $1.4 \mathrm{GHz}(620 \pm 60 \mathrm{mJy})$ from the continuum end channels of the SGPS HI data and at $843 \mathrm{MHz}(680 \pm$ $80 \mathrm{mJy}$ ) from data of the Molonglo Survey (Green et al. 1999). We added two flux density measurements from the literature at $5 \mathrm{GHz}(520 \mathrm{mJy}$, Haynes et al. 1979) and at $4.85 \mathrm{GHz}$ (474 mJy, Wright et al. 1994) (Fig. 10). Since no error was given for the Haynes et al. (1979) value we weighted both fluxes equally. The error bars plotted in Fig. 10 represent $10 \%$ of the flux density. A weighted least-squares fit gives a spectral index of $\alpha=-0.17 \pm 0.07$, that we interpret as arising from optically thin thermal plasma.

The observed diameter of $\mathrm{Wd} 1$ in our $1.4 \mathrm{GHz}$ data is 3.5 at a resolution of $2^{\prime}$. This results in an actual diameter of $3^{\prime}$. This agrees with its appearance in the $843 \mathrm{MHz}$ Molonglo Survey (Green et al. 1999). The observed peak of the radio continuum emission is about $24 \mathrm{~K}$. Correcting for the synthesised beam, the $\mathrm{H}$ II region has a peak brightness temperature of $33 \mathrm{~K}$ at $1.4 \mathrm{GHz}$. The observed brightness temperature $T_{\mathrm{B}}$ at frequency $v$ is related to the electron temperature $T_{\mathrm{e}}$ inside the $\mathrm{H}$ II region and the optical depth $\tau$ by:

$T_{\mathrm{b}}(v)=T_{\mathrm{e}}\left(1-\mathrm{e}^{-\tau(v)}\right)$,

where the optical depth at frequency $v$ is given by:

$\tau(v)=8.235 \times 10^{-2}\left(\frac{T_{\mathrm{e}}}{\mathrm{K}}\right)^{-1.35}\left(\frac{v}{\mathrm{GHz}}\right)^{-2.1}\left(\frac{E M}{\mathrm{pc} \mathrm{cm}^{-6}}\right)$.

(e.g. Rohlfs \& Wilson 2004). Here EM is the emission measure related to the path length $l$ through the H II region and the electron density $n_{\mathrm{e}}$ inside it by:

$E M=\int n_{\mathrm{e}}^{2} \mathrm{~d} l$

With an electron temperature of $T_{\mathrm{e}}=8000 \pm 2000 \mathrm{~K}$ and a path length of $3.5 \mathrm{pc}\left(3^{\prime}\right.$ at $\left.3.9 \mathrm{kpc}\right)$ through the $\mathrm{H}$ II region, we estimate an optical depth of $0.0041_{-0.0008}^{+0.0014}$ and an emission measure of $19000_{-6000}^{+8000} \mathrm{pc} \mathrm{cm}^{-6}$. Hence, $n_{\mathrm{e}}=74 \pm 13 \mathrm{~cm}^{-3}$ and the total mass of the ionized gas inside the $\mathrm{H}$ II region is $53 \pm 9 M_{\odot}$. The frequency at which this emission becomes optically thick (i.e. $\left.\tau_{v}=1\right)$ is $\approx 100 \mathrm{MHz}$.
Alternatively, the electron density of the HII region can be calculated from the observed flux density. For an optically thin Maxwellian plasma, the flux $S_{v}$ is given by:

$S_{v}=5.7 \times 10^{-56} T_{\mathrm{e}}^{-0.5} g_{\mathrm{ff}} E_{V} d^{-2} \mathrm{mJy}$,

where $g_{\mathrm{ff}}$ is the Gaunt-factor defined by:

$g_{\mathrm{ff}}=\frac{\sqrt{3}}{\pi}\left(17.7+\ln \left(\frac{T_{\mathrm{e}}^{1.5}}{v}\right)\right)$

and $E_{V}$ is the volume emissivity:

$E_{V}=\int n_{\mathrm{e}}^{2} \mathrm{~d} V$

The radio spectrum in Fig. 10 indicates that the emission is optically thin over the entire observed frequency range. Using the flux density at $843 \mathrm{MHz}$, we derive an electron density of $n_{\mathrm{e}}=65 \pm 7 \mathrm{~cm}^{-3}$ and a total ionized mass of $M=47 \pm 5 M_{\odot}$. This is in excellent agreement with the value derived from the observed brightness temperature.

\subsection{Wd 1 and its neutral environment}

In Sect. 3, two small (B1 and B2) and one large (B3) bubble in the HI data were described. We believe these are related to $\mathrm{Wd} 1$. The determination of the mass of atomic material in these bubbles is difficult since these objects, in particular bubble B3, seems to merge with the surrounding ISM in the HI images (Figs. 6 and 7). Bubble B1 and B2 together contain a mass of about $300 M_{\odot}$ with an error of at least $50 \%$, assuming the $\mathrm{HI}$ is optically thin and the mass ratio between hydrogen and helium is 10:3. The inner edge of bubble B1 seems to be just outside the Wd $1 \mathrm{H}$ II region (see Fig. 6) giving a diameter of about $5 \mathrm{pc}$ at a distance of $3.9 \mathrm{kpc}$. At the same distance, bubble B2 has an extent of about $18 \times 10$ pc. McClure-Griffiths et al. (2002) defined the dynamic age of a stellar wind bubble that expands as $\propto t^{0.3}$ by:

$t_{6}=0.29 \frac{R}{v_{\exp }}$

where $t_{6}$ is the dynamic age of the bubble in Myr, $R$ the radius in pc, and $v_{\text {exp }}$ the expansion velocity in $\mathrm{km} \mathrm{s}^{-1}$. For bubble B2 the maximum expansion radius calculated from the position of $\mathrm{Wd} 1$ is about $10 \mathrm{pc}$. If we use an expansion velocity of $5 \mathrm{~km} \mathrm{~s}^{-1}$ for B2, determined for bubble B1, we derive a dynamic age of about $600000 \mathrm{yr}$ for B2. Since its structure in velocity space (see Fig. 6) implies a somewhat higher expansion velocity than that for bubble B1 and the radius is a maximum expansion radius, this age represents an upper limit.

Crowther et al. (2006) determined an age of 4.5-5.0 Myr for Wd 1, based on the ratio of WR stars to red and yellow hypergiants. Clearly, bubbles B1 and B2 cannot be the stellar wind bubble produced in the early evolution of $\mathrm{Wd} 1$; they are simply too young. Both B1 and B2 could have been created after the last supernova explosion pushed away all material inside the stellar wind bubble. The recent discovery of the X-ray pulsar CXO J164710.2-455216 inside Wd 1 implies that at least one supernova explosion occured inside $\mathrm{Wd} 1$ (Muno et al. 2006). In this case we speculate that B1 and B2 originate from the only source of matter and energy left after the last supernova occurred, the combined winds and mass loss of the cluster stars. In that scenario, the gas in the shells of those bubbles must consist mostly of recombined material previously 
ejected by the stars in their winds, since the supernova shock wave should have removed any remaining material in the ambient medium. This would imply that the last supernova explosion happened less than $600000 \mathrm{yr}$ ago. Additionally, the ionized material inside the $\mathrm{H}$ II region would also be material ejected by the stars in their winds. The total mass of the ionized gas plus the B2 $\mathrm{HI}$ shell is $\sim 350 M_{\odot}$. If we assume these structures are $500000 \mathrm{yr}$ old we require 70 stars with an average mass-loss rate of $10^{-5} M_{\odot} \mathrm{yr}^{-1}$ for each star, which is highly feasible for the stellar population in $\mathrm{Wd} 1$.

We suspect that the impact of the original stellar wind bubble, created by the members of $\mathrm{Wd} 1$ in their early stage of evolution, is represented by bubble B3 (Fig. 7). Its diameter is about $50 \mathrm{pc}$ at a distance of $3.9 \mathrm{kpc}$. The location of $\mathrm{Wd} 1$ in the lower right corner of this feature is likely the result of a highly structured environment. To the South, away from the Galactic plane, the density is expected to decrease in which case the winds of the members of Wd 1 pushed the material away from the Galactic plane into the Galactic halo. To the West we find a dense cloud complex that impeded free expansion, whereas to the East the winds of the stars pushed material into the cloud centered at the G340.2 - $0.2 \mathrm{H}$ II region complex. In Fig. 8 we can identify three bright compact radio sources that belong to this complex at positions $(l, b)$ : $(340.3,-0.2),(340.1,-0.15)$, and (340.1, $-0.25)$. These are all sources with far infrared colours characteristic of ultra-compact $\mathrm{H}$ II regions, which are known to be regions of OB star formation (Bronfman et al. 1996). This indicates that the G340.2-0.2 H II region complex is younger than Wd 1 and its formation may well have been triggered by the interaction of the early $\mathrm{Wd} 1$ stellar wind or a supernova explosion with the clouds in which G340.2-0.2 is embedded.

To the North of bubble B3 we find a shell that might still be expanding. Since this shell is the only dynamic feature we observe, we use its distance of $45 \mathrm{pc}$ from Wd 1 as the expansion radius. We do not see any expansion of B3 in the velocity slices of our H I data. If we assume that the dynamic age of bubble B3 equals the assumed age of $\mathrm{Wd} 1$, an expansion velocity of about $3 \mathrm{~km} \mathrm{~s}^{-1}$ is implied, which is a reasonable value. Bubble B3 is certainly much older than B2 and B1, because it is many times as large and a low velocity is indicated by the absence of any visible feature in the velocity slices. Unfortunately we cannot estimate the mass of the bubble because of the confusion with the emission regions to the east and the west.

\section{Summary}

Based on H I observations from the SGPS, we have established that Wd 1 is in the Scutum-Crux arm of the Galaxy. We find three large bubbles in which $\mathrm{Wd} 1$ is located at a radial velocity of $-55_{-26}^{+9} \mathrm{~km} \mathrm{~s}^{-1}$. Using a flat rotation model of the Galaxy and adopting a galacto-centric distance of $7.6 \mathrm{kpc}$ (Eisenhauer et al. 2005 ) and a velocity of $214 \pm 7 \mathrm{~km} \mathrm{~s}^{-1}$ for the Sun around the Galactic Centre, we transpose the radial velocity of the bubble features to a distance of $3.9 \pm 0.7 \mathrm{kpc}$ at a Galacto-centric radius of about $4.2 \mathrm{kpc}$. We are confident in our derived distancevelocity calibration in this direction, since it predicts to within $\pm 4 \mathrm{~km} \mathrm{~s}^{-1}$ the velocity of the Tangent point and the velocity of the HI gas in the Sagittarius Arm outside the Solar Circle on the far side of the Galactic Centre. Moreover, this is very encouraging since it is believed that the position of $\mathrm{Wd} 1$ is in the region of the Galactic bar.

A distance of $3.9 \pm 0.7 \mathrm{kpc}$ is somewhat less than the earlier estimate of $4.7 \pm 1.1 \mathrm{kpc}$ based on the absolute magnitude calibration of WR stars (Crowther et al. 2006), but very similar to the $4.0 \pm 0.3 \mathrm{kpc}$ determined by Brandner et al. (2005) from initial analysis of the photometric detection of the main and premain sequence populations in $\mathrm{Wd} 1$. Though the uncertainty on these estimates is relatively large $(\sim 10-25 \%)$, a weighted average of these values gives us confidence that the distance to $\mathrm{Wd} 1$ is $\sim 4 \mathrm{kpc}$. Until the final analysis of the photometric observations described in Brandner et al. (2005) is available, we argue that this represents the best distance estimate to $\mathrm{Wd} 1$.

A study of the $\mathrm{HI}$ images from the vicinity of $\mathrm{Wd} 1$ revealed three bubble-like features B1, B2, and B3. We believe that bubbles B1 and B2 are the result of the stellar winds of the members of $\mathrm{Wd} 1$ and contain recombined wind material. We argue bubble B3 represents the stellar wind bubble created in the early history of $\mathrm{Wd} 1$. The formation of the $\mathrm{H}$ II region complex G340.2-0.2 which is embedded in the eastern edge of B3 may have been triggered by the interaction of the $\mathrm{Wd} 1$ wind with the dense clouds in this area. If this is true, the Wd 1 G340.2-0.2 pair would be a nice example of sequential star formation.

Acknowledgements. We wish to thank Naomi McClure-Griffiths for her help with the SGPS HI and continuum data. We also wish to thank Tom Landecker for careful reading of the manuscript. The Dominion Radio Astrophysical Observatory is a National Facility operated by the National Research Council Canada.

\section{References}

Benjamin, R. A., Churchwell, E., Babler, B. L., et al. 2005, ApJ, 630, L149 Brandner, W., Clark, S., \& Waters, R. 2005, in Protostars and Planets V, 8344 Bronfman, L., Nyman, L.-A., \& May, J. 1996, A\&AS, 115, 81

Burton, W. B. 1972, A\&A, 19, 51

Clark, J. S., \& Negueruela, I. 2002, A\&A, 396, L25

Clark, J. S., Fender, R. P., Waters, L. B. F. M., et al. 1998, MNRAS, 299, L43

Clark, J. S., Negueruela, I., Crowther, P. A., \& Goodwin, S. P. 2005, A\&A, 434, 949

Cordes, J. M., \& Lazio, T. J. W. 2002, ArXiv Astrophysics e-prints

Crowther, P. A., \& Dessart, L. 1998, MNRAS, 296, 622

Crowther, P. A., Hadfield, L. J., Clark, J. S., Negueruela, I., \& Vacca, W. D. 2006, MNRAS, 372, 1407

Eisenhauer, F., Genzel, R., Alexander, T., et al. 2005, ApJ, 628, 246

Feast, M., \& Whitelock, P. 1997, MNRAS, 291, 683

Figer, D. F., McLean, I. S., \& Morris, M. 1999, ApJ, 514, 202

Figer, D. F., Najarro, F., Gilmore, D., et al. 2002, ApJ, 581, 258

Foster, T. \& MacWilliams, J. 2006, ApJ, 644, 214

Green, A. J., Cram, L. E., Large, M. I., \& Ye, T. 1999, ApJS, 122, 207

Haynes, R. F., Caswell, J. L., \& Simons, L. W. J. 1979, Austral. J. Phys. Astrophys. Suppl., 48, 1

McClure-Griffiths, N. M., Dickey, J. M., Gaensler, B. M., \& Green, A. J. 2002, ApJ, 578, 176

McClure-Griffiths, N. M., Dickey, J. M., Gaensler, B. M., et al. 2005, ApJS, 158, 178

Muno, M. P., Clark, J. S., Crowther, P. A., et al. 2006, ApJ, 636, L41

Piatti, A. E., Bica, E., \& Claria, J. J. 1998, A\&AS, 127, 423

Reid, M. J., \& Brunthaler, A. 2004, ApJ, 616, 872

Rohlfs, K., \& Wilson, T. L. 2004, Tools of radio astronomy, Tools of radio astronomy, 4th rev. and enl., ed. K. Rohlfs, \& T. L. Wilson (Berlin: Springer) Russeil, D. 2003, A\&A, 397, 133

Stil, J. M., Taylor, A. R., Dickey, J. M., et al. 2006, AJ, 132, 1158

Taylor, A. R., Gibson, S. J., Peracaula, M., et al. 2003, AJ, 125, 3145

Taylor, J. H., \& Cordes, J. M. 1993, ApJ, 411, 674

Wright, A. E., Griffith, M. R., Burke, B. F., \& Ekers, R. D. 1994, ApJS, 91, 111 